\title{
Osimertinib as first-line therapy in advanced NSCLC: a profile of its use
}

\author{
Lesley J. Scott ${ }^{1}$ \\ Published online: 6 July 2018 \\ (C) Springer Nature 2018, corrected publication 2018
}

\begin{abstract}
Osimertinib (Tagrisso ${ }^{\circledR}$ ) is an oral, CNS-active, third-generation epidermal growth factor receptor (EGFR) tyrosine kinase inhibitor (TKI) that selectively inhibits EGFR TKI-activating mutations over wild-type EGFR in patients with advanced nonsmall cell lung cancer (NSCLC), including the T790M mutation that often underlies acquired resistance to earlier generation EGFR TKIs. Relative to standard of care first-generation EGFR TKIs (erlotinib or gefitinib) as first-line treatment of EGFR activating mutation-positive advanced NSCLC, osimertinib significantly prolongs median progression-free survival (PFS), with separation of the Kaplan-Meier PFS survival curves evident by the first assessment timepoint of 6 weeks. Osimertinib prolongs PFS relative to standard EGFR TKI therapy in all prespecified groups, irrespective of the EGFR mutation present at study entry and presence of CNS metastases at study entry. Overall survival data are not yet mature. Osimertinib has a generally manageable tolerability profile.
\end{abstract}

\section{Adis evaluation of osimertinib as first-line therapy} in advanced NSCLC

Potent, irreversible EGFR TKI of certain mutant EGFR forms [L858R, exon 19 deletion (ex19del), and T790M], with minimal activity against wild type EGFR

Prolongs PFS relative to erlotinib or gefitinib in patients with advanced NSCLC, including those with CNS metastases, and irrespective of the EGFR mutation status (L858R or ex19del)

At the time of data cutoff, overall survival data not yet mature

Generally manageable tolerability profile, with most adverse events being of mild to moderate intensity
Lesley J. Scott

dtp@adis.com

1 Springer, Private Bag 65901, Mairangi Bay, 0754 Auckland, New Zealand

\section{What is the rationale for using osimertinib in NSCLC?}

In recent years, a better understanding of the pathophysiology and numerous oncogenic drivers associated with cancer, such as non-small cell lung cancer (NSCLC), has led to the development of targeted antineoplastic agents that have revolutionized cancer management and improved clinical outcomes [1-3]. Key oncogenic drivers of NSCLC, with NSCLC accounting for $>80 \%$ of lung cancers, involve mutations in the tyrosine kinase domain of the epidermal growth factor receptor (EGFR) gene, with these activating/ sensitizing mutations occurring in $\approx 10$ to $15 \%$ of Caucasian and $\approx 30$ to $40 \%$ of East Asian lung cancer patients [1-3].

Although targeted therapy with first (erlotinib, gefitinib) and second (afatinib) generation EGFR tyrosine kinase inhibitors (TKIs) represent a significant paradigm shift in the management of NSCLC, these drugs are inevitability associated with the emergence of resistance in almost all patients (typically at a median time of 9-16 months), most commonly the EGFR T790M gatekeeper mutation (accounts for $50-60 \%$ of secondary resistance to first-line EGFR TKI therapy) [1-3]. Insights into the mechanisms underlying resistance to earlier generation EGFR TKIs led to the development of third-generation EGFR TKIs that selectively inhibit both $E G F R$ TKI-activating mutations [e.g. exon 19 deletion (ex19del) and L858R mutations] and the T790M resistance mutation [1-3]. Given the minimal inhibition of 
wild-type EGFR TKI by third-generation EGFR TKIs, these compounds may also reduce the toxicities associated with earlier generation EGFR TKIs [1].

Oral osimertinib (Tagrisso ${ }^{\circledR}$ ) is the first and, currently, only third-generation EGFR TKI indicated for the treatment of NSCLC. This review focuses on its use as first-line therapy in patients with locally advanced and metastatic NSCLC whose tumours have EGFR ex19del or exon 21 L858R mutations (i.e. sensitizing/activating mutations). Its use as the first and currently only third-generation EGFR TKI approved to treat EGFR TKI-experienced patients with metastatic EGFR T790M mutation-positive NSCLC has been previously reviewed [4] and is beyond the scope of this article.

\section{For whom is osimertinib indicated?}

Osimertinib is approved in several countries, including those of the EU [5] and the USA [6], for first-line treatment in patients with locally advanced [5] or metastatic [5, 6] NSCLC whose tumours have EGFR ex19del or exon 21 L858R mutations [6] (have activating EGFR mutations [5]). In many countries, osimertinib is also approved for the treatment of patients with locally advanced or metastatic EGFR T790M mutation-positive NSCLC whose disease has progressed on or after EGFR TKI therapy. Table 1 provides a summary of the prescribing information for osimertinib as approved in the EU [5] and USA [6]. Consult local information for further details.

\section{How does osimertinib work?}

Osimertinib potently and irreversibly inhibits the tyrosine kinase activity of certain mutant EGFR forms [L858R, exon 19 deletion (ex 19del) and T790M] at $\approx 9$-fold lower concentrations than wild-type EGFR (i.e. exhibits minimal activity against wild type EGFR) [4, 6]. Of the two pharmacologically active metabolites, AZ7550 exhibited similar potency and selectivity to osimertinib and AZ5104 was a more potent inhibitor of ex19del and T790M mutant $(\approx 8$-fold $)$ and wild-type ( $\approx 15$-fold) EGFR than the parent drug $[4,6]$. In preclinical in vitro and in vivo studies, osimertinib exhibited potent, sustained antitumour activity [4, 6], including in mouse mutant EGFR (ex19del, L858R, L858R/T790M and ex 19del/T790M) NSCLC xenograft models and mouse mutant EGFR (L858R and L858R/T790M) transgenic lung adenocarcinoma models [4]. Osimertinib crosses the bloodbrain barrier and inhibits the growth of CNS metastases in animal models [4]. Osimertinib may potentially delay the emergence of resistance, as observed in an in vitro study in which the emergence of resistance in PC9 cells with the
EGFR ex 19del mutation was delayed with osimertinib compared with other EGFR TKIs [7, 8].

Overtime, as with earlier generation EGFR TKIs, the development of resistance to osimertinib is inevitable [9, 10]. Acquired resistance to osimertinib potentially involves several mechanisms, including the development of C797S resistance mutations at the kinase binding site (primary mechanism), activation of other pathways (e.g. via HER2 and $C M E T$ amplification) and small cell transformation [4, 9].

Clinically significant cardiovascular events may potentially occur with osimertinib therapy, with the QTc interval prolongation of osimertinib $80 \mathrm{mg}$ once daily evaluated in 210 treatment-experienced patients with EGFR T790M-positive NSCLC [5, 6]. A concentration-dependent QTc interval prolongation of $14 \mathrm{~ms}$ was predicted with the $80 \mathrm{mg}$ dose of osimertinib [5, 6].

\section{What is the pharmacokinetic profile of osimertinib?}

Osimertinib exhibits linear pharmacokinetics, with the median peak plasma concentration attained $6 \mathrm{~h}$ after oral administration [6]. Steady-state plasma concentrations of osimertinib are reached after 15 days, with once daily administration resulting in a threefold accumulation of the drug. Osimertinib is primarily metabolized via oxidation (predominantly CYP3A4) and dealkylation in vitro, with two pharmacologically active metabolites (AZ7550 and AZ5104) identified in plasma after oral administration. At steady state, exposure to each of these active metabolites was $\approx 10 \%$ of the exposure to osimertinib. The primary route of elimination of osimertinib is in the faeces (68\%), with $14 \%$ eliminated in the urine and $<2 \%$ eliminated as unchanged drug [6].

\section{What is the efficacy of first-line osimertinib in NSCLC?}

First-line therapy with oral osimertinib $80 \mathrm{mg}$ once daily was more effective than standard of care EGFR TKIs (i.e. erlotinib or gefitinib) in the pivotal randomized, double-blind, multinational FLAURA trial in 556 adults with previously untreated EGFR mutation-positive, advanced NSCLC [11].

At randomization in FLAURA, $\approx 38 \%$ of patients were male, the median age of patients was 64 years, $\approx 98.5 \%$ had adenocarcinoma, 95\% had metastatic disease and 5\% locally advanced disease, 63\% had an EGFR ex19del mutation and $37 \%$ an EGFR L858R mutation, $\approx 21 \%$ had CNS metastases, and $\approx 41$ and $\approx 59 \%$ of patients had a WHO performance status of 0 and 1 [11]. Treatment was continued until 
Table 1 Summary of prescribing information of oral osimertinib (Tagrisso ${ }^{\circledR}$ ) in locally advanced [5] and metastatic [5, 6] NSCLC in the EU [5] and USA [6]

What are the approved indications for osimertinib?

First-line treatment

EU: Adult patients with locally advanced or metastatic NSCLC with activating EGFR mutations

USA: Patients with metastatic NSCLC whose tumours have EGFR exon19 deletions or exon 21 L858R mutations, as detected by an FDA-approved test

Following EGFR TKI therapy

EU: Adult patients with locally advanced or metastatic EGFR T790M mutation-positive NSCLC

USA: Patients with metastatic EGFR T790M mutation-positive NSCLC, as detected by an FDAapproved test, whose disease has progressed on or after EGFR TKI therapy

How is osimertinib available and how should it be administered?

Availability
Dosage
Duration of treatment
Administration in patients who have
difficulty swallowing

Tablets containing 40 or $80 \mathrm{mg}$ of osimertinib

$80 \mathrm{mg}$ once daily, taken without regard to food

Continue until disease progression or unacceptable toxicity

Immerse tablet in 50 [5] or 60 [6] $\mathrm{mL}$ of non-carbonated water

Stir until tablet is dispersed into small pieces (does not fuly dissolve) and swallow immediately

Rinse container with half a glass [5] or 120-240 mL [6] of water and immediately drink

Administration via a nasogastric tube

Disperse tablet in $15 \mathrm{~mL}$ of non-carbonated water, and then use another $15 \mathrm{~mL}$ of water to transfer any residue to the syringe.

Administer this $30 \mathrm{~mL}$ as per nasogastric tube instructions, with water flushes $(\approx 30 \mathrm{~mL})$

How should osimertinib be used in special populations?

Elderly patients (age $\geq 65$ years)

No dosage adjustment required

Patients with renal impairment

Mild, moderate or severe impairment: no dosage adjustment required

End-stage renal disease: caution advised [5] or no recommended dose [6] (lack of data)

Patient with hepatic impairment

Mild or moderate: no dosage adjustment required

Severe impairment: caution advised [5] or no recommended dose [6] (lack of data)

Paediatric patients

Efficacy and safety have not been established

What precautions should be taken with regard to the risk of potential embryo-foetal /infant toxicity?

Female patients of child-bearing potential and male patients with a partner of child-bearing potential

Pregnant women

Women who are breast feeding
Advise of potential risk of embryo-foetal toxicity [5, 6]; only use if benefits outweigh the risks [5]

Female patients: advise them to use effective contraception during treatment and for 6 [6] or $\geq 8$ [5] weeks after the final dose

Male patients: advise them to use effective contraception during treatment and for 4 months after the final dose

Advise of the potential risk for foetal harm [5, 6]; only use if the benefits outweigh the risks [5]

Do not breastfeed during treatment [5, 6] and for 2 weeks after the final dose [6]

What other special warnings/precautions pertain/monitoring requirements to the use of osimertinib?

Interstitial lung disease/pneumonitis

QTc interval prolongation

Cardiomyopathy

Keratitis
Permanently discontinue treatment in patients diagnosed with these conditions

Patients with a history or predisposition to QTc interval prolongation or who are taking medications that are known to prolong the QTc interval: monitor ECGs and electrolytes

Patients with cardiac risk factors: conduct cardiac monitoring, including LVEF assessment

Patients with signs and symptoms of keratitis: promptly refer patients to an ophthalmologist

What are the potential clinically relevant drug interactions between osimertinib and other drugs?

Strong CYP3A4 inducers (e.g. rifampin)

BCRP substrates (e.g. rosuvastatin)
Avoid concomitant use [5] (if possible [6]); if not possible [6], $\uparrow$ osimertinib to $160 \mathrm{mg}$ once daily Resume osimertinib $80 \mathrm{mg}$ once daily 3 weeks after discontinuing the strong CYP3A4 inducer

Monitor for adverse drug reactions of the BCRP substrate (osimertinib $\uparrow$ exposure to such drugs)

Unless otherwise indicated, data pertain to both the EU and the USA

$B C R P$ breast cancer resistance protein, $C Y P$ cytochrome P450, EGFR epidermal growth factor receptor, $L V E F$ left-ventricular ejection fraction, $N S C L C$ non-small cell lung cancer, TKI tyrosine kinase inhibitor, $\uparrow$ increase(s) 
disease progression, unacceptable tolerability or withdrawal of consent, with a median duration of treatment exposure at the cutoff date of 16.2 months in the osimertinib group and 11.5 months in the standard EGFR TKI group. After RECIST-defined disease progression, a first anticancer therapy was initiated in 82 of 279 patients in the osimertinib group and 129 of 277 patients in the standard EGFR TKI group ( 48 of these patients in the standard EGFR TKI group crossed over to osimertinib and 7 received osimertinib outside of the trial as second-line therapy) [11].

In the overall full analysis set (FAS) of FLAURA, osimertinib significantly $(p<0.001)$ prolonged median progression-free survival (PFS) compared with standard EGFR TKI treatment, with a reduction in the risk of disease progression or death of $54 \%$ at the time of data cutoff (primary outcome) [Table 2] [11]. The separation of the osimertinib and standard EGFR TKI PFS Kaplan-Meier curves had occurred by the time of the first assessment at 6 weeks. At the time of data cutoff, 136 and 206 patients in the osimertinib and standard EGFR TKI groups had experienced a RECISTdefined progression or death event. Prespecified subgroup analyses for PFS were consistent with the findings in the FAS analysis, with PFS favouring [i.e. hazard ratio $(\mathrm{HR})<1$, with 95\% CI not crossing 1] osimertinib over standard EGFR TKI therapy across all subgroups, including in patients with or without known or treated CNS metastases at study entry, in Asian and non-Asian patients, and irrespective of the type of mutation present at baseline (Table 2) [11].

There were generally no between-group differences for secondary outcomes in FAS analyses, including for the ORR, disease control rate and median time to response (Table 2) [11]. Osimertinib recipients experienced a significantly better median best percentage change in target lesion size than standard EGFR TKI recipients ( -54.7 vs $-48.5 \%$; $p=0.003$ ); best percentage change in target lesion size was defined as the maximum decrease from baseline or minimum increase from baseline in the absence of a decrease [11].

At the time of data cutoff, overall survival data were not yet mature (25\% maturity reached), with death occurring in 21\% (58 patients) of osimertinib recipients and 30\% (83 patients) of standard EGFR TKI recipients by 18 months (HR for death 0.63 ; 95\% CI 0.45-0.88; $p=0.007$; not significant in this interim analysis) [11]. Consistent with these promising immature overall survival data, the significant PFS benefit with osimertinib over standard EGFR TKI therapy was maintained for the median time-to-event postprogression endpoints of time to first subsequent therapy or death (23.5 vs 13.8 months; HR 0.51 ; 95\% CI $0.40-0.64$; $p<0.0001)$, median time from randomization to second progression on subsequent treatment (not yet reached vs 20.0 months; HR 0.58 ; 95\% CI $0.44-0.78 ; p<0.001)$ and

\section{Table 2 Efficacy of oral osimertinib as first-line treatment for advanced NSCLC with EGFR mutations in the FLAURA trial [11]}

\begin{tabular}{|c|c|}
\hline Outcome & Results \\
\hline Comparators (no. of pts) & Osimertinib vs EGFR-TKI (279 vs 277) \\
\hline \multirow{7}{*}{$\begin{array}{l}\text { Median progression-free survival } \\
\text { in the FAS (primary outcome) and } \\
\text { subgroup analyses }\end{array}$} & In the FAS of 556 pts $^{\mathrm{a}}: 18.9^{*}$ vs 10.2 months [HR 0.46 (95\% CI $\left.0.37-0.57\right)$ ] \\
\hline & In 116 pts with CNS metastases at study entry: $15.2 *$ vs 9.6 months [HR 0.47 (95\% CI $0.30-0.74$ )] \\
\hline & In 440 pts without CNS metastases at study entry: $19.1 *$ vs 10.9 months [HR 0.46 (95\% CI $0.36-0.59$ )] \\
\hline & In 347 Asian pts: HR 0.55 (95\% CI 0.42-0.72) \\
\hline & In 209 non-Asian pts: HR 0.34 (95\% CI 0.23-0.48) \\
\hline & In 349 pts with $E G F R$ exon 19 deletions at study entry: HR 0.43 (95\% CI $0.32-0.56$ ) \\
\hline & In 207 pts with EGFR L885R mutation at study entry: HR 0.51 (95\% CI 0.36-0.71)] \\
\hline \multicolumn{2}{|l|}{ Secondary outcomes in the FAS } \\
\hline Median overall survival & Not yet reached (data were not yet mature at data cut-off; data maturity $25 \%$ ) \\
\hline Objective response rate & $80(95 \%$ CI 75-85) vs 76\% (95\% CI 70-81) \\
\hline Disease control rate ${ }^{\mathrm{a}}$ & 97 (95\% CI 94-99) vs 92\% (95\% CI 89-95) \\
\hline Median duration of response & $17.2(95 \%$ CI $13.8-22.0)$ vs 8.5 months (95\% CI 7.3-9.8) \\
\hline Median time to response & 6.1 (95\% CI 6.0-6.1) vs 6.1 weeks (95\% CI not calculable) \\
\hline
\end{tabular}

Pts were stratified by EGFR mutation status and race, and randomized to receive osimertinib $80 \mathrm{mg}$ once daily or a standard of care oral EGFR TKI (i.e. gefitinib $250 \mathrm{mg}$ once daily or erlotinib $150 \mathrm{mg}$ once daily) until disease progression, unacceptable side effects or withdrawal of consent. Results presented are from the data cutoff of Jun 2017 (136 and 206 events of progression or death had occurred in the osimertinib and EGFR TKI groups)

$E G F R$ epidermal growth factor receptor, $F A S$ full analysis set, $H R$ hazard ratio, pts patients, $T K I$ tyrosine kinase inhibitor

${ }^{*} p<0.001$ vs EGFR TKI

${ }^{\text {a } P t s ~ w h o ~ h a d ~ a ~ c o m p l e t e ~ r e s p o n s e, ~ p a r t i a l ~ r e s p o n s e ~ o r ~ s t a b l e ~ d i s e a s e ~ l a s t i n g ~} \geq 6$ weeks before any disease-progression event 
median time to second subsequent therapy (not yet reached vs 25.9 months; HR 0.60 ; 95\% 0.45-0.80; $p<0.001$ ) [12].

In a prespecified subgroup analysis of FLAURA in patients with documented CNS metastases by neuroradiology (blinded independent central neuroradiology review), osimertinib prolonged PFS compared with standard EGFR TKI therapy [median CNS PFS not reached (95\% CI 16.5, not calculable) vs. 13.9 months (95\% CI 8.3, not calculable); $n=61$ and 67 in CNS FAS], corresponding to a CNS PFS HR of 0.48 (95\% CI 0.26-0.86; nominal $p=0.014)$ [13]. The confirmed CNS objective response rates (ORR) in the osimertinib and standard EGFR TKI group were $57 \%$ and $40 \%$, corresponding to an odds ratio of 2.0 (95\% CI 1.0-1.41; $p=0.053)$. The median CNS duration of response in the osimertinib group was not reached $(95 \%$ CI 11.9, not calculable) and in the standard EGFR TKI group was 14.4 months (95\% CI 8.3-18.7) [13].

There was no between-group difference with regard to changes from baseline to 9 months in key patient-reported outcomes, all of which improved in both treatment groups, with improvements in cough symptoms being clinically relevant [14].

\section{Other clinical data}

The data from FLAURA are supported by evidence from the expansion phase of the phase 1/2 AURA trial in EGFR-TKI treatment-experienced patients with EGFR T790M mutations, which also enrolled two first-line cohorts of EGFR TKI-naive patients with EGFR mutation-positive NSCLC [15]. In the cohort of 30 patients receiving the approved dosage of osimertinib $80 \mathrm{mg}$ once daily, the ORR was $67 \%$, the disease control rate was $93 \%$, and the median duration of response was 19.3 months [15]. In the cohort of 30 patients receiving osimertinib $160 \mathrm{mg}$ once daily, $87 \%$ had an ORR, 100\% achieved disease control, the median PFS was 22.1 months and the median response duration was 16.7 months; corresponding results in the pooled cohorts were $77 \%, 97 \%, 20.5$ months and 18 months [15].

\section{What is the tolerability profile of osimertinib?}

Osimertinib had a generally manageable tolerability as first-line therapy in patients with advanced NSCLC in the FLAURA trial (FAS population) [11]. In the osimertinib and standard EGFR TKI group, 13 and $18 \%$ of patients permanently discontinued treatments because of an adverse event (AE). AEs leading to dose interruptions occurred in $25 \%$ of osimertinib recipients and $24 \%$ of standard EGFR TKI recipients and AEs resulting in dose reductions occurred in
4 and $5 \%$ of patients, respectively. Grade 3 or 4 treatmentemergent AEs occurred in $34 \%$ of osimertinib recipients and $45 \%$ of standard EGFR TKI recipients. The most common (i.e. incidence $\geq 2 \%$ ) possibly causally-related grade 3 AEs occurring in the osimertinib or standard EGFR TKI groups were decreased appetite ( 2 vs $1 \%$ ), diarrhoea ( 2 vs $2 \%$ ), rash or acne (1 vs 7\%), and aspartate (1 vs $4 \%$ ) or alanine (ALT; $<1$ vs $7 \%$ ) aminotransferase elevations. Four patients ( 1 vs $0 \%$ in the osimertinib group) in the standard EGFR TKI group experienced grade 4 ALT elevations, which was the only possibly causally-related grade $4 \mathrm{AE}$ to occur with an incidence of $\geq 2 \%$ in either group [11].

Overall serious AEs occurred in 22 and $25 \%$ of osimertinib and standard EGFR TKI recipients in FLAURA [11]. No fatal adverse events were considered possibly treatmentrelated in the osimertinib group, with one serious case of diarrhoea considered to be possibly related to standard EGFR TKI therapy. Serious AEs of pneumonia (3\%) and interstitial lung disease (ILD; 1\%) occurred with the same incidence in both groups. Of the treatment-emergent ILD cases, all 11 cases in the osimertinib group were considered resolved or resolving, as were 5 of 6 cases in the standard EGFR TKI group (the other patient was considered not recovered). Serious prolongation of the QT interval was reported in one osimertinib recipient and no standard EGFR TKI recipients, with no fatal cases of torsades des pointes or prolongation of the QT interval occurring in either group. The maximum change from baseline $(411.8$ and $408.0 \mathrm{~ms})$ to week 12 in Fridericia's corrected QT (QTcF) interval in the osimertinib and standard EGFR TKI groups was 17.7 and $10.0 \mathrm{~ms}$, after which time QTcF values generally remained stable in both groups [11].

Precautions should be taken to ameliorate the risk and/ or severity of several potential AEs (Table 1). Warnings and precautions regarding the use of once-daily osimertinib $80 \mathrm{mg}$ were based on pooled data from 1142 patients with EGFR mutation-positive NSCLC participating in FLAURA or in EGFR TKI-experienced patients participating in the AURA clinical trial programme [6]. ILD/pneumonitis occurred in $3.9 \%$ of these patients, with $0.4 \%$ of cases fatal. A QTc interval of $>500 \mathrm{~ms}$ occurred in $0.9 \%$ of patients, with $3.6 \%$ experiencing an increase in QTc of $>60 \mathrm{~ms}$. No QTc-related arrhythmias were reported. Cardiomyopathy events (defined as cardiac failure, chronic cardiac failure, congestive heart failure, pulmonary oedema or decreased ejection volume) occurred in $2.6 \%$ of patients, $0.1 \%$ of which were fatal. Of the 908 patients with a baseline and $\geq 1$ follow-up left ventricular ejection fraction (LVEF) assessment, $3.9 \%$ experienced a decline in LVEF of $\geq 10 \%$ from baseline and to $>50 \%$ LVEF. Of the 1142 patients, keratitis occurred in $0.7 \%$. Based on animal data and its mechanism of action, osimertinib can cause foetal harm when administered to pregnant women [6]. 


\section{What is the current clinical position of first-line osimertinib in advanced NSCLC?}

The third-generation EGFR TKI osimertinib is an effective first-line treatment and has a generally manageable tolerability profile in patients with advanced NSCLC with EGFR TKI-activating mutations. In the pivotal FLAURA trial in previously untreated patients with advanced $E G F R$ TKI-activating mutation-positive NSCLC, osimertinib significantly prolonged median PFS compared with standard of care EGFR TKI treatment with erlotinib or gefitinib. The early separation of the PFS Kaplan-Meier curves for osimertinib and these first-generation EGFR TKIs supports the hypothesis that third-generation EGFR TKIs such as osimertinib may prevent/delay the development of resistance [11], with the development of resistance inevitable with all EGFR TKIs [1-3, 9, 10]. Consistent with results in the overall population, osimertinib also prolonged PFS (vs erlotinib or gefitinib) in all prespecified subgroups of patients, including in patients with CNS metastases [11]; patients with $E G F R$ mutation-positive NSCLC are especially prone to the development of brain metastases [16]. Overall survival data for the FLAURA trial are not yet mature [11]. A limitation of the FLAURA trial was the lack of comparison with the second-generation EGFR TKI afatinib, with no head-to head comparisons of osimertinib and afatinib conducted to date.

Osimertinib is the first and, currently, only third-generation EGFR TKI approved for use in EGFR TKI-naive patients with EGFR activating mutation-positive NSCLC and/or EGFR TKI-experienced patients with metastatic EGFR T790M mutation-positive NSCLC. Based on the findings of FLAURA, US treatment guidelines currently recommend osimertinib as a first-line treatment option in patients with EGFR activating mutation-positive NSCLC [17]. The European Society for Medical Oncology (ESMO) guidelines [18] were published prior to the recent positive opinion given by the European Medicines Agency for the use of osimertinib as first-line treatment in patients with locally advanced NSCLC whose tumours have activating EGFR mutations [19]. US treatment guidelines [17] also recommended osimertinib as second or subsequent-line therapy in patients with T790M acquired resistance to firstor second-generation EGFR TKIs who have progressed on these earlier generation EGFR TKIs, as do ESMO guidelines [18]. The optimal approach/sequence for using EGFR TKIs remains to be fully determined, with results of ongoing trials awaited with interest. One such trial, the open-label phase 2 APPLE trial [20], is designed to primarily evaluate the best strategy for delivering osimertinib (i.e. upfront vs sequential treatment after first-generation EGFR TKI) in NSCLC patients with EGFR mutations, as assessed by PFS at 18 months. Among other secondary endpoints, this trial will also evaluate the time to symptomatic brain metastases in patients with brain metastases at trial entry [20].

Acknowledgements The reviewers of this manuscript included: $A$. Morabito, Thoracic Medical Oncology, Istituto Nazionale Tumori, "Fondazione G. Pascale"-IRCCS, Naples, Italy. During the peer review process, AstraZeneca, the marketing-authorization holder of osimertinib, was offered an opportunity to provide a scientific accuracy review of their data. Changes resulting from comments received were made on the basis of scientific and editorial merit.

\section{Compliance with ethical standards}

Funding The preparation of this review was not supported by any external funding.

Conflicts of interest L.S. is an employee of Adis/Springer, is responsible for the article content and declares no conflicts of interest.

Open Access This article is distributed under the terms of the Creative Commons Attribution-NonCommercial 4.0 International License (http://creativecommons.org/licenses/by-nc/4.0/), which permits any noncommercial use, duplication, adaptation, distribution and reproduction in any medium or format, as long as you give appropriate credit to the original author(s) and the source, provide a link to the Creative Commons license and indicate if changes were made.

\section{References}

1. Barnes TA, O'Kane GM, Vincent MD, et al. Third-generation tyrosine kinase inhibitors targeting epidermal growth factor receptor mutations in non-small cell lung cancer. Front Oncol. 2017;7:113. https://doi.org/10.3389/fonc.2017.00113.

2. Choo JR, Tan C-S, Soo RA. Treatment of EGFR T790M-positive non-small cell lung cancer. Targ Oncol. 2018;13:141-56.

3. Tan CS, Kumarakulasinghe NB, Huang YQ, et al. Third generation EGFR TKIs: current data and future directions. Mol Cancer. 2018;17(1):29.

4. Lamb YN, Scott LJ. Osimertinib: a review in T790M-positive advanced non-small cell lung cancer. Targ Oncol. 2017;12(4):555-62.

5. Tagrisso (osimertinib) 40 and $80 \mathrm{mg}$ film-coated tablets: summary of product characteristics. London: European Medicines Agency; 2018.

6. Tagrisso ${ }^{\circledR}$ (osimertinib) tablets, for oral use: US prescribing Information. Wilmington: Astra Zeneca Pharmacueticals LP; 2018.

7. Liam CK. Osimertinib as first-line treatment of EGFR mutant advanced non-small-cell lung cancer. Transl Lung Cancer Res. 2017;6(Suppl 1):S62-6.

8. Jeong BH, Um SW. First-line osimertinib in patients with EGFRmutated advanced non-small cell lung cancer. Ann Transl Med. 2018;6(3):62. https://doi.org/10.21037/atm.2017.12.30.

9. Wang S, Song Y, Yan F, et al. Mechanisms of resistance to third-generation EGFR tyrosine kinase inhibitors. Front Med. 2016;10(4):383-8.

10. Hirsh V. Turning EGFR mutation-positive non-small-cell lung cancer into a chronic disease: optimal sequential therapy with EGFR tyrosine kinase inhibitors. Ther Adv Med Oncol. 2018;10:1-12. 
11. Soria JC, Ohe Y, Vansteenkiste J, et al. Osimertinib in untreated EGFR-mutated advanced non-small-cell lung cancer. N Engl J Med. 2018;378(2):113-25.

12. Planchard D, Boyer M, Lee JS, et al. Osimertinib vs standard of care (SOC) EGFR-TKI as first-line therapy in patients (pts) with untreated EGFRm advanced NSCLC: FLAURA post-progression outcomes [abstract no. 1280]. J Thorac Oncol. 2018;13(4 Suppl):S72-3.

13. Vansteenkiste J, Reungwetwattana T, Nakagawa K, et al. CNS response to osimertinib vs standard of care (SoC) EGFR-TKI as first-line therapy in patients (pts) with EGFR-TKI sensitising mutations (EGFRm)-postive advanced non-small cell lung cancer (NSCLC): data from the FLAURA study [abstract]. In: European Society of Medical Oncology-Asia Conference. 2017.

14. Leighl N, Karaseva N, Nakagawa K, et al. Patient-reported outcomes from FLAURA: osimertinib versus standard of care (SOC) epidermal growth factor receptor tyrosine kinase inhibitor (EGFR-TKI) in patients with EGFR-mutated advanced non-small cell lung cancer (NSLC) [abstract no. 139PD]. J Thorac Oncol. 2018;13(4 Suppl):S81-S82.
15. Ramalingam SS, Yang JC, Lee CK, et al. Osimertinib as first-line treatment of EGFR mutation-positive advanced non-small-cell lung cancer. J Clin Oncol. 2018;36(9):841-9.

16. Sun JM, Park K. Can we define the optimal sequence of epidermal growth factor receptor tyrosine kinase inhibitors for the treatment of epidermal growth factor receptor-mutant nonsmall cell lung cancer? Curr Opin Oncol. 2017;29(2):89-96.

17. National Comprehensive Cancer Network ${ }^{\circledR}$. Clinical Practice Guidelines in Oncology (NCCN Guidelines ${ }^{\circledR}$ ): non-small cell lung cancer (version 1.2018). Fort Washington: National Comprehensive Cancer Network ${ }^{\circledR}$, Inc; 2017.

18. Novello S, Barlesi F, Califano R, et al. Metastatic non small-cell lung cancer: ESMO clinical practice guidelines for diagnosis, treatment and follow-up. Ann Oncol. 2016;27(Suppl 5):v1-27.

19. Summary of opinion (post authorization): Tagrisso (osimertinib). London: European Medicines Agency; 2018.

20. US National Institutes of Health. Osimertinib treatment on EGFR T790M plasma positive NSCLC patients (APPLE) [NCT02856893]. https://clinicaltrials.gov/ct2/show/NCT0285689 3. Accessed 3 July 2018. 